\title{
Simulating exciton migration and annihilation dynamics in higher order spectroscopies of a molecular trimer
}

\author{
Constantin Heshmatpour ${ }^{1}$, František Šanda ${ }^{2}$, Craig N. Lincoln ${ }^{1}$, Vaclav Perlík ${ }^{2}$, Pavel \\ Malevich $^{1}$, and Jürgen Hauer ${ }^{1,3, *}$ \\ ${ }^{1}$ Photonics Institute, TU Wien, Gußhausstraße 27-29, 1040 Vienna, Austria \\ ${ }^{2}$ Institute of Physics, Faculty of Mathematics and Physics, Charles University, Ke Karlovu 5, Prague \\ 121 16, Czech Republic \\ ${ }^{3}$ Professur für Dynamische Spektroskopien, Fakultät für Chemie, Technische Universität München, \\ Lichtenbergstr. 4, D-85748, Garching b. München, Germany
}

\begin{abstract}
We present simulations of $3^{\text {rd }}$ and $5^{\text {th }}$ order multidimensional time-dependent signals of a molecular trimer. The developed model allows for the simulation of both signal types within one theoretical framework. We therefore extended standard Redfield theory of exciton migration by a new term to include the process of exciton-exciton annihilation.
\end{abstract}

\section{Introduction}

2D electronic spectroscopy (2D-ES) has been successfully used to probe relaxation dynamics of single excitons by resolving $3^{\text {rd }}$ order signals in both emission and excitation frequencies as well as in population times [1]. The most common experiments measure so-called rephasing and non-rephasing signals along $\mathbf{k}_{\mathbf{l}}=\mathbf{k}_{1}-\mathbf{k}_{2}+\mathbf{k}_{3}$ and $\mathbf{k}_{\text {II }}=-\mathbf{k}_{1}+\mathbf{k}_{2}+\mathbf{k}_{3}$, respectively. In this notation, $\mathbf{k}_{1,2,3}$ refers to the directions of the three involved excitation pulses.

Dynamics involving multiple excitons can be studied by another $3^{\text {rd }}$ order signal, called 2Q-2D electronic spectroscopy, and measured along $\mathbf{k}_{\mathrm{III}}=\mathbf{k}_{1}+\mathbf{k}_{2}-\mathbf{k}_{3}$. This technique, however, is experimentally challenging, suffers from pulse related artefacts and measures biexcitonic states only as part of an optical coherence, but not directly in their population decay behavior [2]. Dostal et al. [3] recently suggested that certain $5^{\text {th }}$ order signals, measured along $\mathbf{k}_{\mathrm{I}}=2 \mathbf{k}_{1}-2 \mathbf{k}_{2}+\mathbf{k}_{3}$ and $\mathbf{k}_{\mathrm{v}}=-2 \mathbf{k}_{1}+2 \mathbf{k}_{2}+\mathbf{k}_{3}$, probe decays of biexcitonic states more directly by explicitly populating biexcitonic energy levels.

Here, we present a master equation approach to treat $5^{\text {th }}$ and $3^{\text {rd }}$ order dynamics on an equal footing. The standard Redfield theory of excitonic transport is extended by a new term describing exciton-exciton annihilation (EEA, see section 2). We thus provide a comprehensive theoretical framework for a better understanding of multiple exciton effects, essential for reconstructing annihilation-free spectroscopic signals.

\footnotetext{
*Corresponding author: juergen.hauer@tum.de
} 


\section{Results}

Both $3^{\text {rd }}$ and $5^{\text {th }}$ order signals are modeled in a resonantly coupled molecular complex consisting of three subunits, each possessing two energy levels and an associated transition dipole moment $\boldsymbol{\mu}_{\mathrm{i}}$. A trimer is the simplest aggregate allowing transfer between biexciton states, while retaining a tractable number of multiexcitonic states, as necessary for a detailed theoretical description of the nonlinear optical response. The dynamics inside excitonic manifolds, described by the Frenkel exciton Hamiltonian [4], are modulated by local fluctuations of the excited state energy on each monomeric subunit, accounted for by three harmonic coordinates $\hat{Q}_{i}$. Those coordinates are uncorrelated by assumption and characterized by a single correlation function $<\hat{Q}_{i}(t) \hat{Q}_{i}(0)>$. Relaxation rates between eigenstates of the Frenkel exciton Hamiltonian are calculated by using standard Redfield theory.

This Frenkel exciton model describes migration within excitonic manifolds. However, it neglects biexcitonic effects, such as EEA. The latter is regarded as an incoherent process, where excitation energy is resonantly transferred between two adjacent sites. This transfer stimulates one site back to the ground state whereas the other is promoted to a higher lying state. From there it decays via a phonon-assisted non-radiative process back to the first excited state [4]. EEA is therefore a process which reduces the number excitations inside an aggregate. In other words, it induces a transition between the $\mathrm{n}^{\text {th }}$ manifold and the $(n-1)^{\text {th }}$ manifold. We therefore model EEA as a unidirectional transfer to lower excitonic manifolds by developing an addition to Redfield theory based on a quantum master equation in Lindblad form [4]. Fig. 1(a) illustrates this idea for a trimeric molecular structure. All transitions between exciton manifolds due to EEA are indicated by arrows.

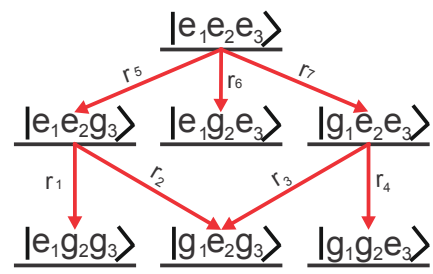

$\underline{\left|g_{1} g_{2} g_{3}\right\rangle}$

(a)

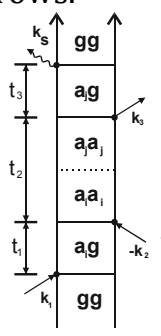

(1)

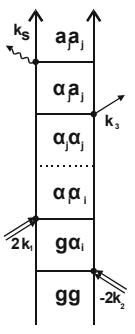

(2)

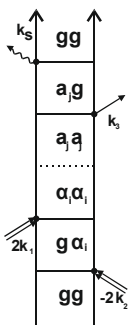

(3)

(b)

Fig.1. Site basis states of a molecular trimer. Arrows indicate unidirectional transfer to lower exciton manifolds due to EEA, with rates $\left\{r_{i}\right\}=\left\{r_{1}, r_{2}, r_{3}, r_{4}, r_{5}=r_{3}, r_{6}=r_{1}+\right.$ $\left.r_{4}, r_{7}=r_{2}\right\}$. (b) Examples for double sided Feynman diagrams contributing to a 3 rd (1) and $5^{\text {th }}(2,3)$ order signal. $|G\rangle:=\left|g_{1} g_{2} g_{3}\right\rangle$ is the ground state wave function, $\left\{\left|a_{i}\right\rangle\right\}$ and $\left\{\left|\alpha_{i}\right\rangle\right\}$ are the eigenstates of the Frenkel exciton Hamiltonian of the $1^{\text {st }}$ and $2^{\text {nd }}$ exciton manifold, respectively. Pathways (1) and (2) represent transport inside excitonic manifolds. Diagram (3) depicts transfer from the $2^{\text {nd }}$ to the $1^{\text {st }}$ exciton manifold, i.e. EEA.

The quantum master equation developed in this work is capable, in combination with the response function formalism [4], to simulate dynamics occurring in both $3^{\text {rd }}$ and $5^{\text {th }}$ order signals of a molecular trimer. Examples of such transfer processes, occurring in both signal types during the waiting time $t_{2}$, are illustrated in Fig. 1(b) with the aid of double sided Feynman diagrams. All of those dynamical processes are captured by the developed quantum master equation. Simulations of spectroscopic signals employing the above described quantum master equation are shown in 
Fig 2(a,b) at $t_{2}=0 \mathrm{fs}$, assuming a linear chain of three transition dipole moments fixed to a head-to-tail arrangement along the longest molecular axis (J-aggregate).

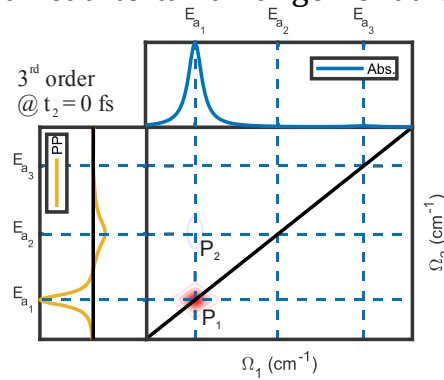

(a)

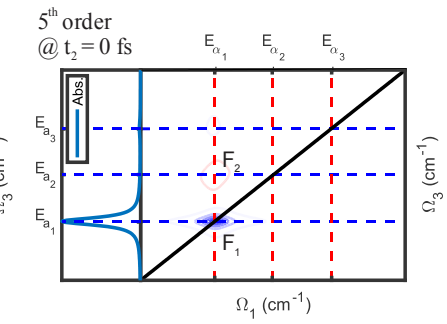

(b)

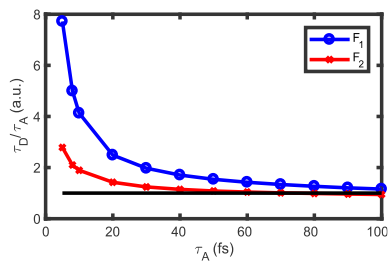

(c)

Fig. 2. Simulated absorption, pump probe and $2 \mathrm{D}$-ES spectra of both $3^{\text {rd }}$ and $5^{\text {th }}$ order signals at $t_{2}=0 \mathrm{fs}$ are depicted in (a) and (b). (c) Plot of the different decay times $\tau_{\mathrm{D}}$ obtained by fitting transients of peak $F_{1}$ and $F_{2}$ along $t_{2}$ by a mono-exponential decay for different annihilation rates $\tau_{\mathrm{A}}$.

The absorption spectrum, depicted in the top panel of Fig. 2(a), shows one main absorption band at the lowest excitonic eigenenergy $E_{a 1}$, typical for J-aggregates. Two main features are observed in the pump probe and $3^{\text {rd }}$ order 2D-ES spectrum shown in Fig 2(a): a strong positive peak $\mathrm{P}_{1}$ and a weak negative peak $\mathrm{P}_{2}$. The positive peak corresponds to ground state bleach (GSB) and stimulated emission (SE) contributions associated with the main absorption band. The negative peak stems from excited state absorption (ESA) pathways. The $5^{\text {th }}$ order spectrum, shown in Fig 2(b), shows a similar two peak structure. Here, positive features are attributed to ESA and negative features to GSB and SE pathways, as the sing is inverted compared to $3^{\text {rd }} 2 \mathrm{D}-\mathrm{ES}$, due to two additional interactions of the dipole operator with the density matrix. The similarity between the two maps arises from the fact that the symmetry of the wave functions in both $1^{\text {st }}$ and $2^{\text {nd }}$ manifold are identical for a J-type configuration. The lowest, symmetric, state therefore carries the most oscillator strength in both cases, giving rise to a single positive and negative feature in both maps. To study the effect of annihilation onto $5^{\text {th }}$ order spectra, all annihilation rates $r_{i}$ were fixed to the same value $r_{a}=1 / \tau_{\mathrm{A}}$ and $\tau_{\mathrm{A}}$ was changed from $10 \mathrm{fs}$ to $100 \mathrm{fs}$ in $10 \mathrm{fs}$ steps. From the obtained $5^{\text {th }}$ order 2D-ES maps, transients were taken at peak positions $F_{1}$ and $F_{2}$ and were fitted with a monoexponential decay. The obtained decay times $\tau_{\mathrm{D}}$, normalized to the input annihilation time $\tau_{\mathrm{A}}$, are plotted in Fig. 2 (c) versus $\tau_{\mathrm{A}}$. For small values of $\tau_{\mathrm{A}}$ a strong deviation is observed, as the dominant rate is given by the relaxation rate inside the $1^{\text {st }}$ manifold. By increasing $\tau_{\mathrm{A}}$ it becomes the dominant timescale and $\tau_{\mathrm{D}}$ therefore gradually approaches $\tau_{\mathrm{A}}$. This corroborates the view that $5^{\text {th }}$ order spectroscopy is a promising tool to understand the general structure of biexciton levels and biexcitonic effects such as EEA, as the $5^{\text {th }}$ order spectrum is distinctly modified by changing the annihilation rate.

\section{References}

1. D.M. Jonas, Annu. Rev. Phys. Chem. 54, 425-63 (2003).

2. V. Perlík, J. Hauer, and F. Šanda, JOSA B 34 (2017).

3. J. Dostal, F. Fennel, F. Koch, S. Herbst, F. Würthner, and T. Brixner, Nat. Commun., 2018. 9(1): p. 2466.

4. V. May, (WILEY-VCH Verlag GmbH \& Co. KgaA, 2011) 